\title{
Data-Dependent Middle-Down Nano-Liquid Chromatography- Electron Capture Dissociation-Tandem Mass Spectrometry: An Application for the Analysis of Unfractionated Histones
}

\author{
Anastasia Kalli, ${ }^{\dagger}$ Michael J. Sweredoski, and Sonja Hess* \\ Proteome Exploration Laboratory, Division of Biology, Beckman Institute, California Institute of Technology, Pasadena, California \\ 91125, United States
}

\section{Supporting Information}

\begin{abstract}
Middle-down mass spectrometry (MS) combined with electron capture dissociation (ECD) represents an attractive method for characterization of proteins and their post-translational modifications (PTMs). Coupling online chromatographic separation with tandem mass spectrometry enables a highthroughput analysis, while improving sensitivity of the electrosprayed peptides and reducing sample amount requirements. However, middle-down ECD has not been thus far coupled with online chromatographic separation. In this work, we examine the

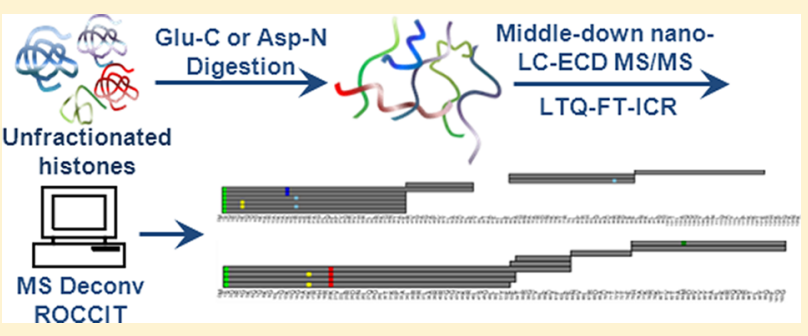
feasibility of coupling middle-down ECD with online nanoflow-liqiud chromatography (nano-LC) for the analysis of large, $>3$ $\mathrm{kDa}$, and highly modified polypeptides in a data-dependent acquisition mode. We evaluate the effectiveness of the method by analyzing peptides derived from Asp- $\mathrm{N}$ and Glu-C digestions of unfractionated histones from calf thymus and acid-extracted histones from HeLa, MCF-7, and Jurkat cells. Our results demonstrate that middle-down ECD is compatible with online chromatographic separation, providing high peptide and protein sequence coverage while allowing precise mapping of PTM sites. The high mass accuracy, obtained by the ICR mass analyzer, for both the precursor and product ions greatly increases confidence in peptide identification, particularly for modified peptides. Overall, for all samples examined, several histone variants were identified and modification sites were successfully localized, including single, multiple, and positional isomeric PTM sites. The vast majority of the identified peptides were in the mass range from 3 to $9 \mathrm{kDa}$. The data presented here highlight the feasibility and utility of nano-LC-ECD-MS/MS for high-throughput middle-down analysis.
\end{abstract}

B ottom-up and top-down are the two main approaches for protein identification and characterization by mass spectrometry. The top-down approach results in complete protein characterization as it allows determination of the intact protein mass providing information about protein isoforms, variants, and combinatorial post-translational modifications (PTMs). When compared to the bottom-up approaches, topdown analysis is less sensitive, requires longer acquisition times, and results in less backbone bond cleavages. ${ }^{1-3}$ In addition, top down is not readily amenable to high-throughput analysis, as it is not easily coupled to online LC separations. ${ }^{2-4}$ Furthermore, chromatographic separation of intact proteins is more challenging than peptide separation. ${ }^{1,2}$ Consequently, the most widely used method for protein identification remains the bottom-up approach, despite several associated limitations, such as very limited information about protein variants or isoforms and combinatorial PTMs. ${ }^{2-4}$ In addition, sample complexity is increased due to the relatively large number of peptides produced following trypsin digestion. ${ }^{2}$

The limitations of bottom-up MS are especially evident when analyzing histones, since histones are highly modified, share a high sequence homology, and have many isoforms and variants. In addition, due to the high content of lysine and arginine residues found in histones, enzymatic digestion with trypsin results in small size peptides. These small size peptides are not well-retained on reverse-phase high-performance liquid chromatography (HPLC) columns, posing a problem for their subsequent analysis by mass spectrometry (MS $)^{3,5}$ and provide very limited information about PTMs occurring in combination. $3,4,6$

A compromise between bottom-up and top-down analysis is the middle-down approach in which peptides $>3000 \mathrm{Da}$ are analyzed. ${ }^{2,7,8}$ These can be produced by enzymes that cleave less frequently than trypsin, such as Arg-C, Asp-N, and Glu-C or by microwave-accelerated acid digestion. ${ }^{7}$ The larger peptides produced by the middle-down approach can reveal information about protein isoforms, variants, and combinations of PTMs, while resulting in a higher confidence in protein identification. ${ }^{1,3,8,9}$ As a consequence, middle-down approaches are particularly appealing for but not limited to the characterization of histones and their PTMs. It should be pointed out that until very recently, the middle-down approach had similar limitations to the top-down approach. Due to recent advances in instrumentation, the sample throughput, sensitivity, and

Received: October 23, 2012

Accepted: February 28, 2013

Published: February 28, 2013 
chromatographic separation are now comparable to that obtained by the bottom-up approach, ${ }^{1,8}$ although automated middle-down LC-MS/MS is far less widespread than the bottom-up approach.

Previously, middle-down LC-collision induced dissociation (CID)-MS/MS was used to analyze Lys-C digests of HeLa-S3 nuclear proteins on an FT-ICR mass spectrometer. ${ }^{10}$ In this analysis, the peptide masses ranged from 1.2 to $15.5 \mathrm{kDa}$, with the majority of the identified peptides being below $3 \mathrm{kDa}^{10}$ Nevertheless, several peptides above $3 \mathrm{kDa}$ were successfully identified and characterized. Fenselau and co-workers used an automated middle-down CID approach for the analysis of the ribosomal proteome. ${ }^{7}$ Automated middle-down CID was also applied for the characterization of complex polyubiquitin chains. ${ }^{11}$

A technique complementary to $\mathrm{CID}$ is electron capture dissociation (ECD) ${ }^{12}$ which results in the formation of $c$ - and $z$-type product ions, instead of $b$ and $y$ ions produced in CID. For several applications, ECD and the related technique, electron transfer dissociation (ETD) ${ }^{13}$ offer significant advantages over CID. For instance, ECD results in more extensive fragmentation ${ }^{12,14-17}$ and consequently in higher peptide sequence coverage and retention of labile PTMs ${ }^{18-32}$ compared to CID. In addition, ECD is very efficient in sequencing highly modified and large polypeptides, ${ }^{33,34}$ rendering it a suitable technique for histone analysis by middle-down MS.

ECD of large polypeptides requires averaging several single scans to obtain spectra with high signal intensity, increasing the average acquisition time and thus far, middle-down ECD has traditionally been performed off-line to allow enough time for data acquisition. Garcia et al. used middle-down ECD to characterize the modification patterns of fractionated, Glu-Cdigested $\mathrm{H} 3.2$ and $\mathrm{H} 3.3$ from different rat tissues. ${ }^{35,36}$

In contrast to data-dependent middle-down ECD, datadependent bottom-up ECD has been employed in several analyses. ${ }^{15,37-42}$ For instance, LC-ECD-MS/MS was used to determine the occupancy rates of phosphorylation sites in human $\alpha_{\mathrm{s1}}$-casein ${ }^{42}$ and novel glycosylation sites of flagellin $\mathrm{A}$ from Campylobacter jejuni. ${ }^{40}$

To reduce analysis time, improve sensitivity, and facilitate analysis of histones and their PTMs, it would be beneficial to couple data-dependent middle-down ECD with online chromatographic separation. However, due to the longer acquisition time required for ECD experiments, middle-down ECD has not yet been coupled with on-line LC. Here, we seek to examine and evaluate the feasibility and applicability of datadependent middle-down ECD for analysis of unfractionated histones and their PTMs. We fully exploit the high mass accuracy and high resolution provided by the FT-ICR mass spectrometer by analyzing both the precursor and fragment ions in the ICR mass analyzer. High-mass accuracy is extremely important for high-confidence identifications of histone modifications as many of these modifications have the same nominal mass and modified residues may have nearly the same mass as unmodified residues. Furthermore, for middle-down analysis, the high resolution of the FT-ICR mass analyzer is essential to resolve and allow determination of the high charge states of the product ions.

Glu-C and Asp-N digests of unfractionated histones from calf thymus and acid-extracted total histones from Jurkat, HeLa, and MCF7 cell lines were used to examine and evaluate the applicability and effectiveness of middle-down ECD coupled to online chromatographic separation operated in a data-dependent acquisition mode. The results obtained suggest that the middle-down nano-LC-ECD-MS/MS approach facilitates high-throughput analysis of unfractionated histones and reveals global modification profiles and localization of PTMs.

\section{EXPERIMENTAL SECTION}

Sample Preparation. Histones from calf thymus, HeLa, Jurkat, and MCF-7 cells were digested either with Glu-C or Asp-N. Detailed description of the sample preparation is available in the Supporting Information.

Nanoflow Liquid Chromatography Electron Capture Tandem Mass Spectrometry (Nano-LC-ECD-MS/MS). All experiments were performed on a nanoflow liquid chromatography system, EASY-nLC II coupled to a 7T LTQ FT-ICR Ultra mass spectrometer, equipped with a nanoelectrospray ion source (all from Thermo Fisher Scientific).

For the EASY-nLC II system, solvent A consisted of $94.8 \%$ $\mathrm{H}_{2} \mathrm{O}, 5 \% \mathrm{ACN}$, and $0.2 \%$ formic acid and solvent $\mathrm{B}$ consisted of $19.8 \% \mathrm{H}_{2} \mathrm{O}, 80 \% \mathrm{ACN}$, and $0.2 \%$ formic acid. For the LCECD-MS/MS experiments, $1.0 \mu \mathrm{g}(5 \mu \mathrm{L})$ of unfractionated Asp-N or Glu-C digested peptides were directly loaded at a flow rate of $500 \mathrm{~nL} / \mathrm{min}$ onto a $16 \mathrm{~cm}$ analytical HPLC column (75 $\mu \mathrm{m}$ ID) packed in-house with ReproSil-Pur $\mathrm{C}_{18} \mathrm{AQ} 3 \mu \mathrm{m}$ resin (120 ̊ pore size, Dr. Maisch $\mathrm{GmbH}$, Ammerbuch, Germany). The column was enclosed in a column heater operating at $40{ }^{\circ} \mathrm{C}$. After $30 \mathrm{~min}$ of loading time, the peptides were separated with a 90 min gradient at a flow rate of $350 \mathrm{~nL} /$ min. The gradient was as follows: $0-5 \%$ solvent B ( $1 \mathrm{~min}), 5-$ $35 \% \mathrm{~B}(80 \mathrm{~min})$, and $100 \% \mathrm{~B}$ (9 $\mathrm{min})$.

The LTQ-FT-ICR was operated in the data-dependent acquisition mode to automatically alternate between a full scan $(\mathrm{m} / z$ range $300-1700)$ and subsequent ECD-MS/MS scans of the three most abundant ions. Both the precursor and product ions were analyzed in the ICR cell. Full MS spectra were acquired with a resolution of 50000 at $\mathrm{m} / z$ 400, with an automatic gain control (AGC) target value of $1 \times 10^{6}$ ions and maximum ion injection time of $700 \mathrm{~ms}$. Dynamic exclusion was enabled with a repeat count of 1 , a repeat duration of $30 \mathrm{~s}$, an exclusion list size of 500, and an exclusion duration of $60 \mathrm{~s}$. The exclusion time was based on the LC gradient used. Precursor ions were isolated in the linear ion trap with an isolation width of $6 \mathrm{~m} / z$ and transferred to the ICR cell for the ECD experiments. ECD was performed with an indirectly heated dispenser cathode (Heatwave, Watsonville, CA, USA). The AGC target value was set at $1 \times 10^{6}$ ions and the maximum ion injection time at $1500 \mathrm{~ms}$. Each ECD spectrum comprised four microscans. Samples were analyzed at different electron energies and/or irradiation times (from 0.21 to $2.21 \mathrm{eV}$ and 50 to $70 \mathrm{~ms}$ ). ECD mass spectra were acquired with a resolution of 25000 or 50000 at $\mathrm{m} / z$ 400. In total, five LCECD-MS/MS analyses were performed for each sample. The exact electron energy, irradiation time, and resolution used for each ECD experiment are given in Tables S1 of the Supporting Information. MS and MS/MS spectra were acquired in the profile mode. Data acquisition was controlled by Xcalibur 2.0 and Tune Plus 2.2 (Thermo Fisher Scientific).

Data Analysis. Mass spectrometric raw data files were deconvoluted with MS Deconv. ${ }^{43}$ An in-house developed search engine (http://roccit.caltech.edu) was used for peptide and protein identifications with a false discovery rate of less than $1 \%$ at both the peptide and protein levels. Description of the search engine is given in the Supporting Information. The 
deconvoluted .mgf files from the 5 LC-ECD-MS/MS analyses, collected at different electron energies and irradiation times, were analyzed together. For the precursor ions, the initial mass tolerance was set to $15 \mathrm{ppm}$. A high mass accuracy filter was then used to further reject peptide spectra matches that have an abnormally high mass error. After filtering, the effective precursor mass tolerance was typically within $5 \mathrm{ppm}$. For the product ions, the mass tolerance was set at $15 \mathrm{ppm}$. Both $c^{\bullet} / c^{\prime}$ and $z^{\bullet} / z^{\prime}$ product ions were searched. Full Glu-C or Asp-N enzymatic specificity was required and up to four missed cleavages were allowed, although only up to two missed cleavages were observed experimentally. Methionine oxidation, acetylation ( $\mathrm{K}$ and $\mathrm{N}$-terminal), methylation ( $\mathrm{K}$ and $\mathrm{R}$ ), dimethylation $(\mathrm{K})$, trimethylation $(\mathrm{K})$, deamidation ( $\mathrm{Q}$ and $\mathrm{N}$ ), and formylation $(\mathrm{K})$ were set as variable modifications. Data were searched against a custom database containing all human (downloaded from UniProt on $10 / 28 / 2011$ ) or bovine (downloaded from UniProt on 01/28/2011) histones and contaminant sequences. Assignments were manually evaluated. A full list of identifications are given in the Supporting Information in .xls format.

\section{RESULTS AND DISCUSSION}

Optimization of middle-down nano-LC-ECD-MS/MS. The longer acquisition time required for ECD and the detection of both precursor and product ions in the ICR cell will result in a longer duty cycle. Therefore, during middledown LC-ECD-MS/MS analysis, fewer peptides will be analyzed. To overcome this limitation, the proteolytic enzymes chosen for middle-down analysis should ideally produce the lower number of peptides for a given sample. As illustrated in Figure S1, in silico Glu-C and Asp-N digestions result in a lower number of proteolytic peptides compared with Arg-C, Lys-C, and trypsin.

The second factor that needs to be considered for middledown analysis is the use of enzymes, which will produce long peptides in the desirable mass range for middle-down analysis ( $>3000 \mathrm{Da}$ ), while producing few peptides having a mass of $<3000 \mathrm{Da}$. Glu-C and Asp-N produce the higher number of peptides having a mass $\geq 3000 \mathrm{Da}$ and the fewer number of peptides with a mass $\leq 3000 \mathrm{Da}$ (Figure S2 of the Supporting Information). Therefore, for middle-down analysis of histones, Glu-C and Asp-N were chosen for enzymatic digestion.

Generally in ECD, highly charged polypeptides require lower energies and irradiation times, whereas peptides at lower charge states fragment more efficiently under higher electron energies and irradiation time. Following Glu-C and Asp-N digestion, the resulting proteolytic peptides have charge states ranging from +2 to +13 and they also cover a very wide range of $m / z$ ratios (from $\sim 300$ to 1200 ). We therefore acquired several LC-ECDMS/MS analyses at different electron energies and irradiation times and examined their effect on the ECD outcome (Figures S3 to S9 of the Supporting Information). Acquiring LC-ECDMS/MS analyses at different experimental conditions allowed for improved ECD fragmentation for a wider range of peptides. In contrast, due to the peptide diversity, a single electron energy and irradiation time setting does not provide optimum fragmentation for all peptides present. It is worth pointing out that achieving optimum ECD fragmentation, that is obtaining high peptide sequence coverage, is essential for precise PTM localization. Therefore, each sample was analyzed under different ECD experimental conditions, and the data files were processed together as detailed in the Experimental
Section. More details on the method optimization are provided in the Supporting Information and Figures S10-S18 of the Supporting Information.

Middle-Down Nano-LC-ECD-MS/MS Analysis. Unfractionated histones from calf thymus, HeLa, Jurkat, and MCF7 cells were digested with Glu-C and Asp-N and subjected to nano-LC-ECD-MS/MS analysis. An overview of the experiment is given in Figure S19 of the Supporting Information. For peptides derived from Glu-C digestion, $79 \%$ of the identified peptides had a mass ranging from 3 to $9 \mathrm{kDa}$ and $21 \%$ have a mass of $<3 \mathrm{kDa}$ (Figure S20A of the Supporting Information). For peptides derived from Asp-N digestion, $73 \%$ of the identified peptides had a mass greater than $3 \mathrm{kDa}$, whereas $27 \%$ had a mass less than $3 \mathrm{kDa}$ (Figure S20B of the Supporting Information).

Figure 1 and Figure S21 of the Supporting Information show representative spectra obtained on a chromatographic timescale by middle-down ECD. Figure 1A shows the spectrum of an 8.9 $\mathrm{kDa}$ polypeptide obtained from $\mathrm{H} 4$, following Asp-N digestion of histones from calf thymus. For this peptide, the ECD sequence coverage was $50 \%$, whereas for the peptide displayed in Figure S21 of the Supporting Information, it was 77\%. Both spectra represent a single MS/MS scan (4 microscans), illustrating that a sufficient number of product ions with good signal intensity and $\mathrm{S} / \mathrm{N}$ ratio can be obtained in a single scan to allow peptide identification. Furthermore, the high peptide sequence coverage obtained is advantageous, as it greatly improves confidence in peptide identification and thus protein identification.

An associated challenge with histone analysis is the differentiation of histone variants, as many histone family members have high sequence homology, exhibiting only small differences in their sequence. For instance, histones H2A 2-A and H2A.1 differ only at amino acids at the positions 16 and 51 . The sequence alignment of these variants and the Asp-N derived peptides analyzed by data-dependent ECD are shown in Figure S22 of the Supporting Information. The complete protein sequence coverage obtained by ECD for both $\mathrm{H} 2 \mathrm{~A} 2-\mathrm{A}$ and H2A.1 allowed differentiation and assignment of both histone variants (Figures S22 and S23 of the Supporting Information).

Equally important to differentiation of histone variants is the identification, characterization, and localization of PTMs. Figure 1B displays the ECD spectrum of the diacetylated and dimethylated N-terminal peptide 1-53 of histone $\mathrm{H} 4$, derived from a Glu-C digestion of histones from MCF-7 cells. This peptide has 8 possible acetylation sites ( $\mathrm{N}$-terminus and 7 Lys residues) and 16 possible sites for dimethylation (7 Lys and 9 Arg residues). The high mass accuracy of both the precursor and product ions allows for confident assignment of the peptide and confirms the presence of three modification sites. Precise localization of these modification sites requires efficient sequencing along the peptide backbone due to the high number of Lys and Arg residues present in the peptide. Following data-dependent ECD, cleavages at 40 out of 52 backbone inter-residue bonds were observed, enabling precise mapping of all three modification sites as illustrated in Figure 2. These modifications are known modification sites for histone H4.

Identification and localization of modification sites can be complicated by the presence of positional isomers (peptides with the same amino acid sequence that are modified at different positions). The presence of such species in histones 

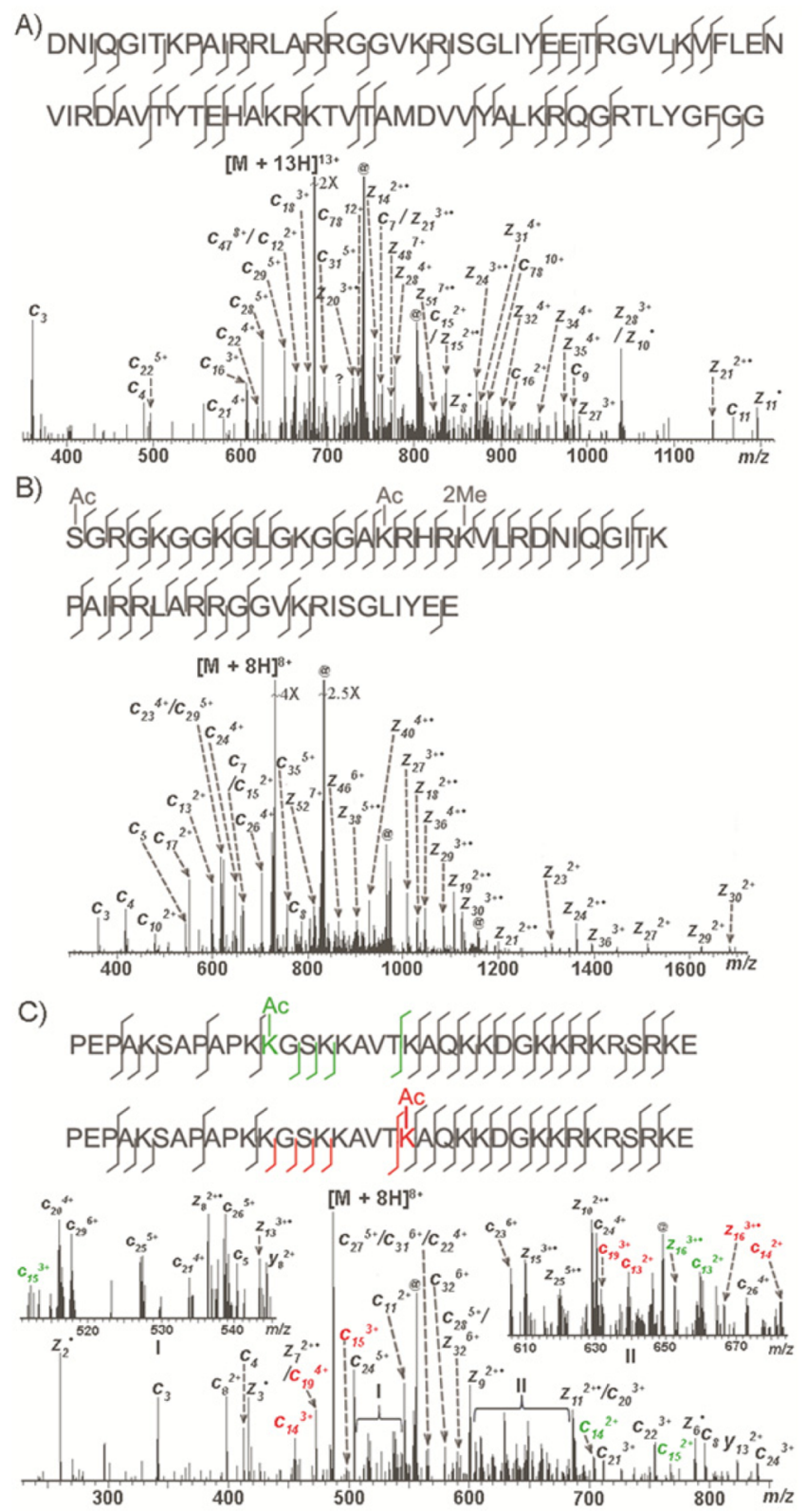

Figure 1. Data-dependent middle-down ECD of (A) peptide 24-102 $(8.9 \mathrm{kDa})$ from $\mathrm{H} 4$, (B) diacetylated and dimethylated peptide 1-53 from histone $\mathrm{H} 4$, and (C) acetylated peptide isomers of $\mathrm{H} 2 \mathrm{~B}$ variants. Peptide 24-102 is from Asp-N digestion of histones from calf thymus. Peptide 1-53 was derived from a Glu-C digestion of histones extracted from MCF-7 cells. Peptides in (C) were derived from a Glu-C digest of Jurkat cells and correspond to the N-terminal tail 1-35 of H2B 1-K (and/or H2B 2-E, H2B 1-J, and H2B 1-C/E/F/G/I). Product ions shown in green correspond to the peptide with an acetylated Lys at position 12, and product ions displayed in red correspond to the peptide with acetylation at Lys-20. Only the major peaks are labeled in the figure. ${ }^{@}=$ charge-reduced species and neutral- or side-chain losses.

has been previously discussed in very few reports. ${ }^{44,45}$ Nicklay et al. reported the presence of two isomeric species of the peptide 1-23 from $\mathrm{H} 4,{ }^{44}$ whereas Bonenfant et al. reported the presence of three positional isomers, with acetylation at Lys-12, Lys-15, and Lys-20 of the peptide 1-25 from H2B variants. ${ }^{45}$ These reports used off-line separation to obtain purified fractions of individual histones, followed by enzymatic digestion of each fraction and subsequent analysis by LC-MS/MS. ${ }^{44,45}$

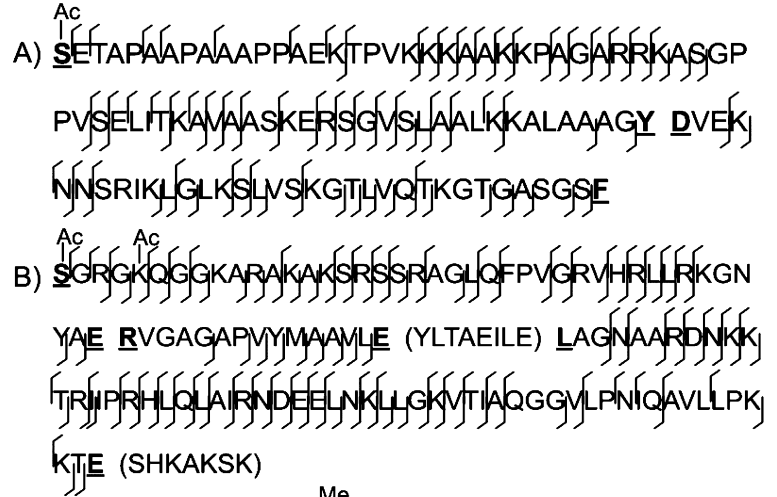

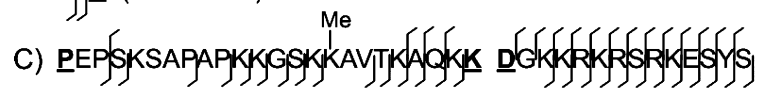

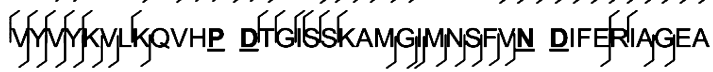

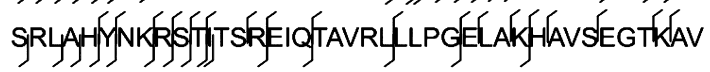
frkyfriss

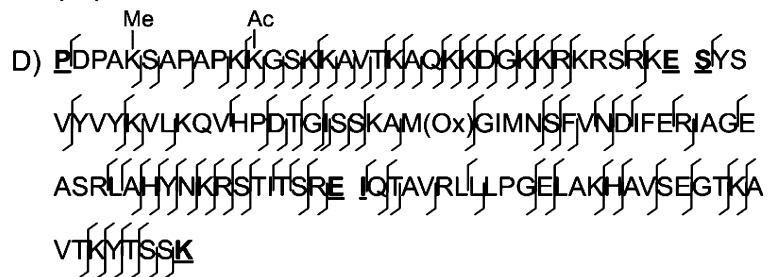

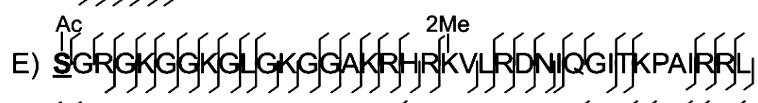

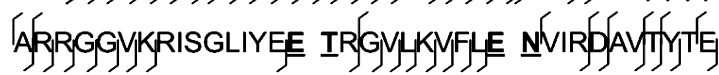

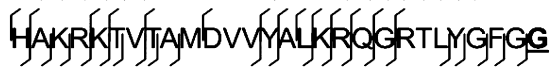

Figure 2. Peptide and protein sequence coverage observed for (A) H1.1, (B) H2A 2-C, (C) H2B 1-N, (D) H2B 1-H, and (E) H4 in middle-down LC-ECD-MS/MS. H1.1 is derived from an Asp-N digestion from histones from calf thymus. H2A 2-C is from a Glu-C digest of histones extracted from MCF-7 cells. H2B 1-N and H2B 1-H are derived from an Asp- $\mathrm{N}$ and Glu-C digest, respectively, from histones from Jurkat cells. $\mathrm{H} 4$ is from a Glu-C digest of histones extracted from HeLa cells. The first and last amino acids of each peptide subjected to fragmentation are shown in underlined and bold fonts. The peptides shown in parentheses in (B) were not detected.

In our analysis, the off-line separation was eliminated and unfractionated histones were digested and subjected to LCECD-MS/MS analysis. Nevertheless, our approach also allowed for characterization of positional isomers as illustrated in Figure 1C. The ECD spectrum of the peptide 1-35 shared by different $\mathrm{H} 2 \mathrm{~B}$ variants (H2B 1-K, H2B 2-E, H2B 1-J, and H2B 1-C/E/ $\mathrm{F} / \mathrm{G} / \mathrm{I}$ ) (Figure 1C) corresponds to a mixed population of two coeluting isomeric peptides. Manual interpretation of the spectrum allowed differentiation and characterization of the two positional isomers. Distinct sets of product ions (colorcoded in Figure 1C) are present for each isomer, one featuring an acetylated Lys residue at position 12 and the other featuring an acetylated Lys at position 20. The same peptide sequence acetylated at Lys-15 was also identified in a different ECD spectrum (Figure S24C of the Supporting Information). These acetylated peptide isomers were also detected in histone $\mathrm{H} 2 \mathrm{~B}$ from HeLa cells (Figure S26C of the Supporting Information). For MCF-7 cells, no modification sites were detected (Figures S28C and S28D of the Supporting Information). 
The peptide and protein sequence coverage obtained for some of the histone variants identified are summarized in Figure 2B. With the exception of histone H2A 2-C (Figure 2), complete protein sequence coverage was observed. Overall, the results summarized in Figure 2 illustrate that the middle-down LC-ECD-MS/MS approach results in high peptide and protein sequence coverage and successful characterization and localization of multiple PTMs sites, rendering this approach an effective method for histone analysis.

It is worth noting that the peptides displayed in Figure 2 were not the only peptide detected for each histone. In fact, overlapping peptides were commonly observed for the majority of the identified histones as shown in Figure 3, which displays all peptides detected for H2A 1-C (Figure 3A), H4 (Figure $3 \mathrm{~B})$, and $\mathrm{H} 2 \mathrm{~B}$ 1-O (Figure 3C). More importantly, this figure summarizes all PTMs observed for $\mathrm{H} 2 \mathrm{~A} 1-\mathrm{C}, \mathrm{H} 4$, and $\mathrm{H} 2 \mathrm{~B}$ 1-O and reveals that information about different combinations of PTMs can be obtained. Analogous results were obtained for all identified histones from the four samples examined and the data summarizing the peptides identified, and combinations of PTMs observed are provided in Figures S24A-S31D of the Supporting Information. It is worth pointing out that obtaining this information from a typical bottom-up approach is extremely challenging due to the small size of the resulting proteolytic peptides.

Table 1 and Tables S2 to S8 (Supporting Information) summarize the identified histone variants, the protein sequence coverage, and the observed PTMs for Jurkat, HeLa, MCF-7 cells, and calf thymus. In summary, for the Glu-C digest of the unfractionated histones from Jurkat cells (Table 1), we identified 25 histone variants and 25 modifications sites. In total, 30 modified peptides were observed, corresponding to 24 uniquely modified peptides. For the vast majority of the histones identified, complete or very high $(>87 \%)$ protein sequence coverage was obtained.

Similar results to those observed for Glu-C, high protein sequence coverage, identification of several histone variants and modifications sites, were obtained for all samples examined (Tables S2-S8 of the Supporting Information). It should be noted that a higher number of histone variants and PTMs can be assessed by off-line prefractionation to obtain purified fractions of individual histone families. Nevertheless, the middle-down nano-LC-ECD-MS/MS method presented here can be equally applied for the analysis of both unfractionated and fractioned samples.

\section{CONCLUSIONS}

We have successfully coupled middle-down ECD with online nanoflow-LC, in a data-dependent fashion, for the analysis of peptides derived from Glu-C and Asp-N digestions of acidextracted unfractionated histones from various cell lines. Our results demonstrate the feasibility and usefulness of datadependent middle-down nano-LC-ECD-MS/MS for the analysis of large (>3000 Da) and highly modified peptides. Overall, high peptide and protein sequence coverage was obtained for all samples examined, and several histone variants and modification sites were identified. Additionally, information about combinatorial PTMs and positional isomers was obtained.

The peptide sequence coverage obtained by the middledown nano-LC-ECD-MS/MS approach was comparable with that observed in middle-down ECD, using static electrospray direct infusion. ${ }^{33,35,36}$ The great advantages of coupling ECD
A.

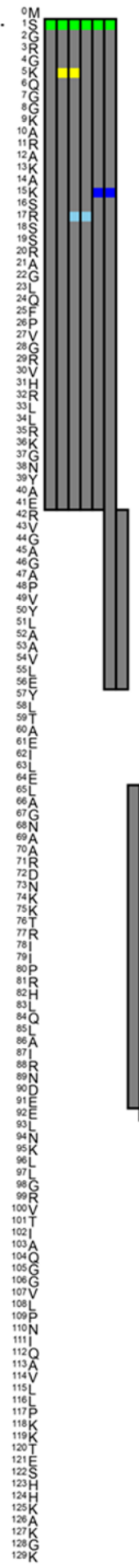

B.

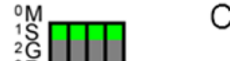

C.

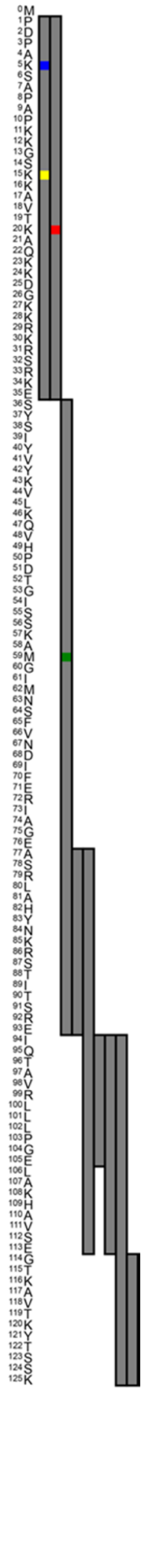

Figure 3. Peptides identified and PTM combinations observed for (A) H2A 1-C from Jurkat cells, (B) H4 from MCF-7 cells, and (C) H2B 1$\mathrm{O}$ from HeLa cells. Peptides were derived from Glu-C digestion. 
Table 1. Summary of Identified Histone Variants, Sequence Coverage Obtained, and Observed PTMs for Histones Extracted from Jurkat Cells Digested with Glu-C ${ }^{a}$

\begin{tabular}{|c|c|c|c|}
\hline $\begin{array}{l}\text { histone } \\
\text { variant }\end{array}$ & $\begin{array}{l}\text { accession } \\
\text { number }\end{array}$ & $\begin{array}{c}\text { protein } \\
\text { sequence } \\
\text { coverage }(\%)\end{array}$ & observed PTMs \\
\hline $\mathrm{H} 4$ & P62805 & 100 & $\begin{array}{c}\text { N-terminal Ac, Lys-16 Ac, } \\
\text { Lys- } 202 \mathrm{Me}\end{array}$ \\
\hline $\mathrm{H} 2 \mathrm{~B} 1-\mathrm{H}$ & Q93079 & 100 & $\begin{array}{l}\text { Lys-5 Me, Lys-12 Ac, } \\
\text { Lys-15 Ac, Lys-20 } 2 \mathrm{Me}\end{array}$ \\
\hline H2B 2-F & Q5QNW6 & 100 & none \\
\hline $\begin{array}{l}\mathrm{H} 2 \mathrm{~B} 1- \\
\mathrm{C} / \mathrm{E} / \mathrm{F} / \mathrm{G} / 1\end{array}$ & P62807 & 100 & $\begin{array}{c}\text { Lys-12 Ac, Lys-15 Ac, } \\
\text { Lys-20 Ac }\end{array}$ \\
\hline $\mathrm{H} 2 \mathrm{~B} 1-\mathrm{K}$ & 060814 & 100 & $\begin{array}{l}\text { Lys-12 Ac, Lys-15 Ac, } \\
\text { Lys-20 Ac }\end{array}$ \\
\hline $\mathrm{H} 2 \mathrm{~B} 1-0$ & P23527 & 100 & $\begin{array}{l}\text { Lys-5 Me, Lys- } 12 \text { Ac, } \\
\text { Lys- } 15 \text { Ac, Lys-20 2Me }\end{array}$ \\
\hline H2B 1-J & P06899 & 100 & $\begin{array}{l}\text { Lys-12 Ac, Lys-15 Ac, } \\
\text { Lys-20 Ac }\end{array}$ \\
\hline H2B 2-E & Q16778 & 100 & $\begin{array}{c}\text { Lys-12 Ac, Lys-15 Ac, } \\
\text { Lys-20 Ac }\end{array}$ \\
\hline $\mathrm{H} 2 \mathrm{~A} 2-\mathrm{C}$ & Q16777 & 95 & $\begin{array}{c}\text { N-terminal Ac, Lys-5 Ac, } \\
\text { Lys-15 Me, Arg-17Me, } \\
\text { Lys-95 For }\end{array}$ \\
\hline H2A.X & P16104 & 94 & N-terminal Ac \\
\hline H2A 1-D & P20671 & 93 & N-terminal Ac, Lys-95 For \\
\hline $\mathrm{H} 2 \mathrm{~A} 1-\mathrm{H}$ & Q96KK5 & 91 & $\begin{array}{c}\text { N-terminal Ac, Lys-5 Ac, } \\
\text { Lys-95 For }\end{array}$ \\
\hline H2A.3 & Q7L7L0 & 87 & N-terminal Ac, Lys-15 Me \\
\hline $\mathrm{H} 2 \mathrm{~A} 1-\mathrm{C}$ & Q93077 & 87 & $\begin{array}{l}\text { N-terminal Ac, Lys-5 Ac, } \\
\text { Lys-15 Me, Arg-17 Me }\end{array}$ \\
\hline H2A 2-B & Q8IUE6 & 87 & $\begin{array}{c}\text { N-terminal Ac, Lys-5 Ac, } \\
\text { Lys-15 Me, Arg-17 Me, } \\
\text { Arg- } 88 \mathrm{Me}\end{array}$ \\
\hline $\mathrm{H} 2 \mathrm{~A} 1-\mathrm{B} / \mathrm{E}$ & P04908 & 87 & N-terminal Ac \\
\hline $\mathrm{H} 2 \mathrm{~A}$ & A6NKY0 & 59 & Lys-99 2Me \\
\hline $\mathrm{H} 3.2$ & Q71DI3 & 55 & none \\
\hline $\mathrm{H} 1.2$ & P16403 & 53 & none \\
\hline $\mathrm{H} 1.5$ & P16401 & 52 & N-terminal Ac \\
\hline H1.4 & P10412 & 51 & none \\
\hline $\mathrm{H} 1.3$ & P16402 & 51 & none \\
\hline H2A.Z & P0C0S5 & 47 & none \\
\hline H2B 3-B & Q8N257 & 39 & Arg-92 Me \\
\hline H1.x & Q92522 & 9 & none \\
\hline
\end{tabular}

${ }^{a}$ Ac $=$ acetylation; $\mathrm{Me}=$ methylation; $2 \mathrm{Me}=$ dimethylation; For $=$ formylation.

with online separation is the increased sensitivity and speed compared to the offline direct infusion approach. Furthermore, with the online nano-LC approach, less sample is required.

In addition, the high mass accuracy employed for both the precursor and product ions allowed for very high-confidence peptide identifications. Taken together, the data presented here, strongly suggests that the middle-down nano-LC-ECD-MS/ MS is an attractive and alternative method for the automated and effective analysis of large and highly modified peptides.

\section{ASSOCIATED CONTENT}

\section{S Supporting Information}

Additional information as noted in text. This material is available free of charge via the Internet at http://pubs.acs.org.

\section{AUTHOR INFORMATION}

\section{Corresponding Author}

*E-mail: shess@caltech.edu. Tel: (626) 395-2339. Fax: (626) 449-4159.

\section{Present Address}

${ }^{\dagger}$ Department of Pathology and Laboratory Medicine, Children's Hospital Los Angeles, Los Angeles, CA 90027, USA.

\section{Notes}

The authors declare the following competing financial interest(s): S.H. is a consultant for Genentech.

\section{ACKNOWLEDGMENTS}

This work was supported by the Betty and Gordon Moore Foundation and the Beckman Institute. We thank Tsui-Fen Chou and Raymond J. Deshaies for providing the HeLa, Jurkat, and MCF-7 cells.

\section{REFERENCES}

(1) Meyer, B.; Papasotiriou, D. G.; Karas, M. Amino Acids 2011, 41, 291-310.

(2) Wu, S. L.; Kim, J.; Hancock, W. S.; Karger, B. J. Proteome Res. 2005, 4, 1155-1170.

(3) Arnaudo, A. M.; Molden, R. C.; Garcia, B. A. Crit. Rev. Biochem. Mol. Biol. 2011, 46, 284-294.

(4) Young, N. L.; DiMaggio, P. A.; Garcia, B. A. Cell. Mol. Life Sci. 2010, 67, 3983-4000.

(5) Garcia, B. A.; Mollah, S.; Ueberheide, B. M.; Busby, S. A.; Muratore, T. L.; Shabanowitz, J.; Hunt, D. F. Nat. Protoc. 2007, 2, 933-938.

(6) Garcia, B. A. Front. Biosci., Scholar Ed. 2009, 1, 142-153.

(7) Cannon, J.; Lohnes, K.; Wynne, C.; Wang, Y.; Edwards, N.; Fenselau, C. J. Proteome Res. 2010, 9, 3886-3890.

(8) Garcia, B. A. J. Am. Soc. Mass Spectrom. 2010, 21, 193-202.

(9) Zhou, H.; Ning, Z. B.; Starr, A. E.; Abu-Farha, M.; Figeys, D. Anal. Chem. 2012, 84, 720-734.

(10) Boyne, M. T.; Garcia, B. A.; Li, M. X.; Zamdborg, L.; Wenger, C. D.; Babai, S.; Kelleher, N. L. J. Proteome Res. 2009, 8, 374-379.

(11) Xu, P.; Peng, J. M. Anal. Chem. 2008, 80, 3438-3444.

(12) Zubarev, R. A.; Kelleher, N. L.; McLafferty, F. W. J. Am. Chem. Soc. 1998, 120, 3265-3266.

(13) Syka, J. E. P.; Coon, J. J.; Schroeder, M. J.; Shabanowitz, J.; Hunt, D. F. Proc. Natl. Acad. Sci. U.S.A. 2004, 101, 9528-9533.

(14) Axelsson, J.; Palmblad, M.; Hakansson, K.; Hakansson, P. Rapid Commun. Mass Spectrom. 1999, 13, 474-477.

(15) Creese, A. J.; Cooper, H. J. J. Am. Soc. Mass Spectrom. 2007, 18, 891-897.

(16) Kruger, N. A.; Zubarev, R. A.; Carpenter, B. K.; Kelleher, N. L.; Horn, D. M.; McLafferty, F. W. Int. J. Mass Spectrom. 1999, 182, 1-5.

(17) Kruger, N. A.; Zubarev, R. A.; Horn, D. M.; McLafferty, F. W. Int. J. Mass Spectrom. 1999, 185, 787-793.

(18) Haselmann, K. F.; Budnik, B. A.; Olsen, J. V.; Nielsen, M. L.; Reis, C. A.; Clausen, H.; Johnsen, A. H.; Zubarev, R. A. Anal. Chem. 2001, 73, 2998-3005.

(19) Håkansson, K.; Chalmers, M. J.; Quinn, J. P.; McFarland, M. A.; Hendrickson, C. L.; Marshall, A. G. Anal. Chem. 2003, 75, 3256-3262.

(20) Kjeldsen, F.; Haselmann, K. F.; Budnik, B. A.; Sorensen, E. S.; Zubarev, R. A. Anal. Chem. 2003, 75, 2355-2361.

(21) Håkansson, K.; Cooper, H. J.; Emmett, M. R.; Costello, C. E.; Marshall, A. G.; Nilsson, C. L. Anal. Chem. 2001, 73, 4530-4536.

(22) Stensballe, A.; Jensen, O. N.; Olsen, J. V.; Haselmann, K. F.; Zubarev, R. A. Rapid Commun. Mass Spectrom. 2000, 14, 1793-1800.

(23) Liu, H.; Håkansson, K. Anal. Chem. 2006, 78, 7570-7576.

(24) Chalmers, M. J.; Kolch, W.; Emmett, M. R.; Marshall, A. G.; Mischak, H. J. Chromatogr., B 2004, 803, 111-120.

(25) Adamson, J. T.; Håkansson, K. J. Proteome Res. 2006, 5, 493501.

(26) Chalmers, M. J.; Quinn, J. P.; Blakney, G. T.; Emmett, M. R.; Mischak, H.; Gaskell, S. J.; Marshall, A. G. J. Proteome Res. 2003, 2, 373-382.

(27) Kelleher, N. L.; Zubarev, R. A.; Bush, K.; Furie, B.; Furie, B. C.; McLafferty, F. W.; Walsh, C. T. Anal. Chem. 1999, 71, 4250-4253. 
(28) Mirgorodskaya, E.; Roepstorff, P.; Zubarev, R. A. Anal. Chem. 1999, 71, 4431-4436.

(29) Kweon, H. K.; Hakansson, K. J. Proteome Res. 2008, 7, 749-755.

(30) Shi, S. D. H.; Hemling, M. E.; Carr, S. A.; Horn, D. M.; Lindh, I.; McLafferty, F. W. Anal. Chem. 2001, 73, 19-22.

(31) Chalmers, M. J.; Hakansson, K.; Johnson, R.; Smith, R.; Shen, J. W.; Emmett, M. R.; Marshall, A. G. Proteomics 2004, 4, 970-981.

(32) Cooper, H. J.; Hakansson, K.; Marshall, A. G. Mass Spectrom. Rev. 2005, 24, 201-222.

(33) Kalli, A.; Hakansson, K. Mol. BioSyst. 2010, 6, 1668-1681.

(34) Mikesh, L. M.; Ueberheide, B.; Chi, A.; Coon, J. J.; Syka, J. E. P.; Shabanowitz, J.; Hunt, D. F. Biochim. Biophys. Acta 2006, 1764, 18111822.

(35) Garcia, B. A.; Siuti, N.; Thomas, C. E.; Mizzen, C. A.; Kelleher, N. L. Int. J. Mass Spectrom. 2007, 259, 184-196.

(36) Garcia, B. A.; Thomas, C. E.; Kelleher, N. L.; Mizzen, C. A. J. Proteome Res. 2008, 7, 4225-4236.

(37) Cooper, H. J.; Akbarzadeh, S.; Heath, J. K.; Zeller, M. J. Proteome Res. 2005, 4, 1538-1544.

(38) Sweet, S. M. M.; Bailey, C. M.; Cunningham, D. L.; Heath, J. K.; Cooper, H. J. Mol. Cell. Proteomics 2009, 8, 904-912.

(39) Savitski, M. M.; Nielsen, M. L.; Zubarev, R. A. Mol. Cell. Proteomics 2005, 4, 1180-1188.

(40) Zampronio, C. G.; Blackwell, G.; Penn, C. W.; Cooper, H. J. J. Proteome Res. 2011, 10, 1238-1245.

(41) Savitski, M. M.; Nielsen, M. L.; Zubarev, R. A. Mol. Cell. Proteomics 2006, 5, 935-948.

(42) Kjeldsen, F.; Savitski, M. M.; Nielsen, M. L.; Shi, L.; Zubarev, R. A. Analyst 2007, 132, 768-776.

(43) Liu, X. W.; Inbar, Y.; Dorrestein, P. C.; Wynne, C.; Edwards, N.; Souda, P.; Whitelegge, J. P.; Bafna, V.; Pevzner, P. A. Mol. Cell. Proteomics 2010, 9, 2772-2782.

(44) Nicklay, J. J.; Shechter, D.; Chitta, R. K.; Garcia, B. A.; Shabanowitz, J.; Allis, C. D.; Hunt, D. F. J. Biol. Chem. 2009, 284, $1075-1085$.

(45) Bonenfant, D.; Coulot, M.; Towbin, H.; Schindler, P.; van Oostrum, J. Mol. Cell. Proteomics 2006, 5, 541-552. 